\title{
Is It Possible To Test The Maximum Repetition For The Inspiratory Muscle? Feasibility, Reliability and a Comparison with the Maximum Dynamic Inspiratory Pressure
}

\author{
Thiago Queiroz Pires, Bruno Prata Martinez, Fábio Santos de Jesus, Saaid Freitas Pires, \\ Mansueto Gomes Neto
}

\begin{abstract}
To analyze the feasibility and use maximum repetition test (MR) as a way to measure inspiratory muscle strength, comparing it with the maximum dynamic inspiratory pressure. A cross-sectional observational study was conducted. Two moments of evaluations were performed, in the first, evaluating the reliability of the MR test, then comparing it with the maximum dynamic inspiratory pressure ( $\mathrm{S}$-index). The MR test was started with $30 \%$ of the $S$-index, with the load being increased by $10 \mathrm{cmH} 2 \mathrm{O}$ in each maneuver, until the concentric failure was registered. A sample consisted of 146 subjects, the CCI for intra-examiner testing was 0.99 , the MR test also demonstrated excellent inter-examiners with an ICC of 0.99. In the comparison between two measures, there was an average difference of $17.7 \pm 11.4 \mathrm{cmH} 2 \mathrm{O}$ (p: $<0.001$ ), despite the high correlation between them.It is feasible and reliable to evaluate the maximum repetition of the inspiratory muscles, the mean values obtained were different from the $S$-Index values.
\end{abstract}

Index Terms - Maximum inspiratory pressure; maximum repetition; inspiratory muscle training; pulmonary function.

\section{INTRODUCTION}

Muscle strength is an important parameter in the evaluation of health outcomes. Studies have shown that lower muscle strength is associated with greater mortality and morbidity [1]-[3].Inspiratory muscle strength is also associated with mobility decline in older people/subjectsregardless lower peripheralmuscle strength and physical activity [4]. In addition, inspiratory muscle weakness is associated with a wide range of adverse health outcomes in chronic diseases [5]-[7].Thus, the ability to accurately determine peripheral or inspiratory muscle strength isclinicallyimportant.

Inspiratory muscle weakness is routinely treated with inspiratory muscle training (IMT), performed using linear load devices, which impose resistance to inspiratory incursion

Thiago Queiroz Pires, Federal University of Bahia, Brazilian Hospital Services Company,Salvador, Brazil,

Bruno Prata Martinez, Federal University of Bahia, State University of Bahia.

Fábio Santos de Jesus, Social organization Irmã Dulce, Reative SpecializedPhysiotherapy.

Saaid Freitas Pires, Santa Izabel Hospital, Reative Specialized Physiotherapy.

Mansueto Gomes Neto,Federal Universityof Bahia. by the patient, however the way of titrating the load for IMT is still very questionable [7]-[8].

For the peripheral muscle strength, measures of the incremental tests are precise and validated. The incremental test determines the maximum load that can be overcome by the skeletal muscle, thus estimating its maximal strength [8]. As for inspiratory muscles, tests performed in clinical practice are important to monitor the improvement or worsening of strength, but they may not be as accurate for titrating the IMT load, since during its execution the individual performs a free inspiratory incursion, without any resistance, only capturing the maximum pressure generated during inspiration, which does not guarantee an ideal load titration during the IMT [8]-[9].Non-invasive measures such as direct inspiratory pressure measurement, using the analog or digital manovacuometer or measure of inspiratory muscle strength as the measurement of maximal dynamic inspiratory pressure (S-index) performed through the Power Breathe K5® device are routinely applied to assess inspiratory muscle strength[9]-[10].These measurements are performed without using an incremental load and estimate only the highest pressure generated in the system, thus it does not represent the maximum load tolerated by the inspiratory muscle, this can lead to inadequate prescribing, especially when this individual performs analogue device training at home, where it usually cannot generate an effective trigger of the device, becoming a placebo training.

Lima et al., identified that incremental tests are little used in clinical practice. They observed that only $22.1 \%$ of the professionals interviewed used an incremental load test to assess inspiratory strength ${ }^{11}$. In addition, those who have already used did not perform the test for a maximal repetition correctly.

On the other hand, the measurement of maximal respiratory muscle strength is also important for respiratory muscle training. Training with maximum repetition zones for skeletal muscles in order to maintain activity until concentric failure already has evidence demonstrating a more effective potential than submaximal training [12]. However, due to the lack of the maximum force measure for the respiratory muscles, the inspiratory muscle strength training can be underestimated. Thus, the absence of an evaluation method that can identify 
Is It Possible To Test The Maximum Repetition For The Inspiratory Muscle? Feasibility, Reliability and a Comparison with the Maximum Dynamic Inspiratory Pressure

the maximum load tolerated by this musculature, makes it impossible to prescribe more precisely the effort to be generated by this muscle group.

In this way, to validate a test that uses incremental load, can be important,because it is a dynamic evaluation, that simulates training tool, allowing greater practical applicability, avoiding failure of device triggering or prescription of ineffective load for inspiratory musclestrength gain training[8].The hypothesis of this study is that an incremental test toassess respiratory muscle strength using maximal repetition test is reliable and viable. Thus, the present study aims to analyze the viability and reliability of the maximal repetition test as a means of measuring inspiratory muscle strength in healthy individuals, comparing it with maximal dynamic inspiratory pressure.

\section{METHODS}

Study design: An observational cross-sectional study was conducted. The inclusion criteria werehealthy volunteers from both sexes required to be between 18 and 60 years old who had no history of pulmonary, cardiac, or neuromuscular disease. Initially we measured age, weight, height, and body mass index (BMI) Body weight was measured using a scale accurate to $100 \mathrm{~g}$. Height was measured by a stadiometer with barefoot subjects. BMI was calculated by dividing body weight $(\mathrm{kg})$ by height squared $\left(\mathrm{m}^{2}\right)$. All the subjects followed a sequence of evaluations based on operational protocol used to this study.

The device used for evaluation was the Power Breathe K5®, monitored using the Breathe Link software, which in its test mode, allows the measurement of the maximum dynamic inspiratory pressure. In training mode, it is possible to set the load manually, with a precise variation of up to $1 \mathrm{cmH} 2 \mathrm{O}$.

Patients were consecutively invited to enter the protocol following the signature of an informed consent. The project was approved by the Institutional Ethics Research Committee.

\section{Procedures}

The evaluations took place in two stages, at first, the objective was to test the reliability of the test of a maximum repetition, being recruited 30 individuals. However, the initial test load of a maximum repetition was titrated to $30 \%$ of the S-index, so, it was necessary to titrate the maximum dynamic inspiratory pressure before starting the $1 \mathrm{MR}$ test. In all measurements, the subject was positioned and instructed to use the Power Breathe K5® mouthpiece connected to the nasal clip to prevent air leakage. Individuals performed a full expiration up to residual volume level, followed by inspiration with greater strength and speed, repeating the maneuver three times, with an interval of two minutes between them, the best measure being considered the S-Index[10]. The maneuvers were performed with the subjects seated in a chair and wearing nose clips following American Thoracic Society (ATS) standards on respiratory muscle testing[13].
For the maximum repetition measurement, an initial load of $30 \%$ of the S-index was stipulated[14]. The participants performed a single maximal inspiration, and it was verified if the effort was able to fire the electronic valve of the device (trigger), also it was recorded the volume of air inspired with this resistance. In subsequent evaluations, if there was a decrease of $10 \%$ of the initial volume (generated with $30 \%$ of the S-index), a cycle execution failure would be detected. Between the series, a two-minute interval was performed, sufficient time to recompose the short-term energy production path (via ATP-CP), as described by Ydfors et al[15]. Then an increase in the load of only $10 \mathrm{cmH}_{2} \mathrm{O}$ was performed (aiming at gradual increase in order to avoid fatigue) and again a maximum inspiration was requested, and the inspiratory cycles lasted until the moment the patient could not trigger the valve again or presented a drop/reduction of more than $10 \%$ in the volume of inspired air. At this time, a decrease in the resistance offered in $01 \mathrm{cmH}_{2} \mathrm{O}$ was initiated, and further maximum incursions were made to each reduction, always respecting the proposed interval, until the moment the patient achieved an effective effort to trigger the valve and maintain the proposed volume. This last load found was titled as the measure of a maximum repetition.

The evaluation of intra- and inter-examiner reliability was performed by two evaluators (A1 and A2) trained in relation to the evaluation procedures. At the beginning of the testing sessions, instructions about the procedure were given in a standardized manner and all measurements were performed by an appropriately trained examiner. Before the measurements, each participant performed 5 maximum inspiratory and 5 maximum expiratory warm-up efforts to familiarize with the procedure. To test intra-examiner reliability, participants were given the guidance of the investigator A 1 and after a minimum interval of 72 hours, they repeated the same procedure. To assess intra-examiner reliability, the investigator A1 assessed the same participants twice.To assess inter-examiner reliability, two investigators (A1 and A2) were blinded to one another's readings. The measurements occurred at the same time of day for each participant. Participants were randomized to define which of the evaluators would first perform the measurement. After the first measurement, the participant evaluated by A1 investigator was evaluated by $\mathrm{A} 2$ and the individual evaluated by $\mathrm{A} 2$ was evaluated by $\mathrm{A} 1$.

The second stage consisted of comparing the values obtained in the test of a maximum repetition with the $S$-index, aiming to identify if there would really be a significant difference between the measures. Thus, over 116 individuals were recruited. All of them performed both tests only once, following the methods described above.A summary of the evaluation methods is shown in figures $\mathrm{S} 1$ and $\mathrm{S} 2$ of the supplement.

Statistical Analyses

Data of continuous variables were analyzed by using measures of central tendency and dispersion and expressed as mean and standard deviation. Categorical or dichotomous variables were analyzed by using measures of frequency. We 
performed Shapiro-Wilk statistical testto evaluate normality for all variables. Intra- and inter-rater reliability were tested using intra-class correlation coefficient (ICC), standard error of measurement, and Bland and Altman analysis. ICCs were calculated with $95 \%$ Cis for all measures of inspiratory muscle performance using a two-way mixed-effects model for single measurements and absolute agreement. An ICC generally implies poor stability for values $<0.40$, fair stability for values between 0.40 and 0.59 , good stability for values between 0.60 and 0.74 and an excellent agreement for values $>0.74^{16}$. Sample size calculation revealed that the appropriate sample size for ICC values $>0.9$ was 11 participants $(\mathrm{w}=0.2$, $\alpha=.05) .30$ participants were recruited to increase the magnitude of the study effect [17].

Bland-Altman plots were also used to determine if any systematic differences across the range of values occurred between the two testing sessions.We used the Bland-Altman method, which includes a scatter plot of the differences between test and retest against their mean, to determine the magnitude of disagreement between tests values. This method also includes the $95 \%$ limits of agreement of differences and the mean difference. To evaluate changes over time in an individual the magnitude of the change has to exceed the inherent variability of the outcome. Thus, if the limits of agreement are very broad and clinically important, it suggests that test/retest could result in a clinically meaningful intra-patient difference[18].

A (p)-value, of 0.05 was considered significant. Statistical analyses were conducted by using the Statistical Package for the Social Sciences, version 21.0 (SPSS Inc., Chicago, IL, EUA). The difference between the means of the S-Index and the MR were analyzed using the Student $\mathrm{T}$ test for paired samples, in which a (p)-value less than 0.05 was considered significant.

\section{RESULTS}

A total study sample consisted of 146 subjects, with mean age of $36.0 \pm 9$ years old; BMI $26.4 \pm 4.6 \mathrm{~kg} / \mathrm{m}^{2}$ and male predominance $(\mathrm{n}=106 ; 72.6 \%)$. Participants characteristics are described in table 1.

\section{Intra- and inter-examiner reliability}

The estimated intra-examiner reliability of the 2-day tests using the ICC showed that the ICC for the intra-examiner test was 0.996 (IC 95\%=0,993-0,998). The MR test also demonstrated excellent inter-examiner reliability with a 0,997 ICC (IC 95\%=0,994-0,999). The results for Intra- and inter-examiner reliability of MR in the study sample are summarized in Table 2.

Difference between maximum repetition and maximum dynamic inspiratorypressure

In the comparison between the two measurements, the average found in the evaluation of the S-Index was $111.8 \pm$ $19.7 \mathrm{~cm} \mathrm{H}_{2} \mathrm{O}$, while in the evaluation of the MR method it was $94.0 \pm 20.7 \mathrm{cmH}_{2} \mathrm{O}$, with an average difference of $17.7 \pm$ $11.4 \mathrm{cmH}_{2} \mathrm{O}(\mathrm{p}:<0.001)$.
Correlation between the two measures and failure detection methods

According to figure 1, the intraclass correlation coefficient (ICC) between the S-Index and MR showed a R $=0.913(95 \%$ $\mathrm{CI}=0.88-0.94)$, with $\mathrm{p}$ value $=0.0001$, which demonstrates a strong correlation for linear relationship between the two variables. When analyzing the precision between the two variables by the Bland-Altman method, an average bias of $17.8 \mathrm{cmH}_{2} \mathrm{O}$ was observed, with lower agreement limits of $-5 \mathrm{cmH}_{2} \mathrm{O}$ and upper agreement of $40.6 \mathrm{cmH}_{2} \mathrm{O}$ (figure 2). The other inter and intra-examiner correlations are shown in figures 3 and 4 .

Regarding the way of detecting the failure, $21.2 \%$ of the participants showed a reduction in the volume of inspired air during the incursion, whereas the majority of the group $(78.8 \%)$ presented a trigger failure during the execution of the test.

\section{DISCUSSION}

The one-repetition maximum test was reproducible as a means of assessing inspiratory muscle strength, with no need to interrupt the test, no adverse events associated with its performance in the study population, and high reliability being feasible and safe. The values of the MR test and the maximum dynamic inspiratory pressure revealed an average difference of $15.9 \%$, with the S-index always being higher. This difference could have been higher because, in this study, the individuals performed the S-Index maneuver for three repetitions, identifying the greatest measure, yet in Silva et. al studypublished after the conclusion of this study, in which it was verified that a previous warm up could potentiate the S-Index, which would possibly increase the disparity between the values found in both methods evaluated[19].

The different values found suggest that not necessarily the highest pressure generated only by the inspiratory muscle will be the load it will support, which may have a direct impact on the changes in the training prescription for this muscle group. Several factors may influence inspiratory muscle capacity, Romer et. al., in 2008, when discussing causal factors of inspiratory muscle fatigue, cited the fact that not necessarily the respiratory muscle needs to be under direct stress to reach its activity limit, however, Arend applied in his study a preheating protocol, with incremental loads, where it was found that previously stimulation of inspiratory muscles increased the value of S-index[20]-[21].Minahan, on the other hand, sought to evaluate whether high-intensity exercise series on an exercise bike could modify the dynamic maximal inspiratory pressure, finding a negative result for this outcome, raising questions about the peripheral muscle interference on inspiratory muscle performance, which reinforces the need to find the maximum load supported for this muscle group, given its high physiological specificity [22].

In 2002, the American Thoracic Society (ATS) published a 
Is It Possible To Test The Maximum Repetition For The Inspiratory Muscle? Feasibility, Reliability and a Comparison with the Maximum Dynamic Inspiratory Pressure

document with guidelines on respiratory muscle function tests, in which the study by Johnson et. al, was cited, describing a protocol, in which a linear load of $10 \mathrm{cmH}_{2} \mathrm{O}$ was established, with increments of $5 \mathrm{cmH}_{2} \mathrm{O}$ every two minutes, and the test was completed when the subject could not trigger the pressure valve for two consecutive breaths[13], [23]. Martyn performed an incremental test starting from a load of $30 \%$ of the maximum static inspiratory pressure, incremented in every two minutes, with the greatest load supported for two minutes established as the maximum resistance[14].However, none of the previously described studies used a load decrease of only $1 \mathrm{cmH}_{2} \mathrm{O}$, after noting the failure, seeking to verify the greatest load overdue in a maximum inspiration. Applying the MR test can identify the maximum load point supported, facilitating the application of inspiratory muscle training (IMT). For example, Fry, in 2007, applied a 10-week inspiratory muscle training protocol in patients with amyotrophic lateral sclerosis, guiding the initial load by the static MIP, using a load of $30 \%$ of the value obtained in the test, however, for load increment, scales of subjective perception of effort were used, since one of the goals of a training program is to find a load that keeps the principle of active overload, however, without generating muscle damage or simply placebo effect, when the individual cannottrigger the device and ends up not performing the training session, this point of work, corroborates with the idea that the application of Incremental tests can facilitate not only the initial training prescription but also assist in the progression of loads[24].

The present study focused on the adaptation of amethodto assess peripheral muscle strength to inspiratory muscle strength, understanding that it is possible to increase load until reaching maximum repetition of this musculature, however, this test may have a prolonged time of application in relation to the usual ones, which becomes a limiting point. An equation that can predict a baseline value is required so that the test can be started from this point, reducing the time required for the evaluation. Other limiting point of the study is the fact that the study was performed with healthy people, and the individual needed to have agood level of cognition preserved, directly impacting its applicability for mechanically ventilated patients, who generally do not have full ability to learn and perform the technique. The findings lead to new/further research, now expanding to specific groups, noting their results.

\section{CONCLUSION}

It is feasible and reliable to evaluate the maximum repetition of inspiratory muscles in healthy individuals, the mean values obtained were different from the values of the maximum dynamic inspiratory pressure.

\section{REFERENCES}

[1]. Celis-Morales CA, Welsh P, Lyall DM, Steell L, Petermann F, Anderson $\mathrm{J}$ et al. Associations of grip strength with cardiovascular, respiratory, and cancer outcomes and allcause mortality: prospective cohort study of half a million UK Biobank participants.BMJ. 2018;361: k1651. doi: 10.1136/bmj. k1651.
[2]. Cooper R, Kuh D, Hardy R, Mortality Review Group. FALCon and HALCyon Study Teams Objectively measured physical capability levels and mortality: systematic review and meta-analysis. BMJ 2010;341:c4467. 10.1136/bmj.c4467

[3]. Leong DP, Teo KK, Rangarajan S, et al. Prospective Urban Rural Epidemiology (PURE) Study investigators Prognostic value of grip strength: findings from the Prospective Urban Rural Epidemiology (PURE) study. Lancet 2015; 386:266-73. 10.1016/S0140-6736(14)62000-6.

[4]. Buchman AS, Boyle PA, Wilson RS, Leurgans S, Shah RC, Bennett DA.Respiratory muscle strength predicts decline in mobility in older persons.Neuroepidemiology. 2008;31(3):174-80. doi: 10.1159/000154930.

[5]. Singer J, Yelin EH, Katz PP,Sanchez G, Iribarren C, Eisner MD, et al Respiratory and skeletal muscle strength in chronic obstructive pulmonary disease: impact on exercise capacity and lower extremity function. J CardiopulmRehabilPrev 2011; 31: 111-119.

[6]. Polkey MI1, Lyall RA2, Yang K3, Johnson E3, Leigh PN4, Moxham J.Respiratory Muscle Strength as a Predictive Biomarker for Survival in Amyotrophic Lateral Sclerosis.Am J RespirCrit Care Med. 2017 Jan 1;195(1):86-95. doi: 10.1164/rccm.201604-08480C.

[7]. Human A, Corten L, Jelsma J, Morrow B.Inspiratory muscle training for children and adolescents with neuromuscular diseases: A systematic review.NeuromusculDisord. 2017 Jun;27(6):503-517. doi: 10.1016/j.nmd.2017.03.009.

[8].Zanini A, Aiello M, Cherubino F, et al.The one repetition maximum test and the sit-to-stand test in the assessment of a specific pulmonary rehabilitation program on peripheral muscle strength in COPD patients. Int J ChronObstructPulmonDis 2015; 10: 2423-2430.

[9].Ohara DG, Pegorari MS, Oliveira Dos Santos NL, de Fátima Ribeiro Silva C, Monteiro RL, Matos AP, Jamami M. Respiratory muscle strength as a discriminator of sarcopenia in community-dwelling elderly: a crosssectional study. J Nutr Health Aging. 2018; 22:952-958.

[10].Lee KB, Kim MK, Jeong JR, Lee WH. Reliability of an electronic inspiratory loading device for assessing pulmonary function in poststroke patients. Med SciMonit. 2016; 22:191-196.

[11].Lima SC, Ribeiro NS, Oliveira NF, Miranda MC, Britto RR, Montemezzo D. Identificação de equipamentos e procedimentos utilizados por fisioterapeutas brasileiros para testes de endurancemuscular inspiratória.FisioterPesqui. 2018;25(3):269-277.

[12]. Ahtiainen, J.P.; Hakkinen, K. Strength Athletes Are Capable to Produce Greater Muscle Activation and Neural Fatigue During High-Intensity Resistance Exercise Than Nonathletes. The Journal of Strength \& Conditioning Research. 2009; 23(4): 1129-1134.

[13].American Thoracic Society, European Respiratory Society. ATS/ ERS Statement on Respiratory Muscle Testing. Am J RespirCritCare Med. 2002;166(4):518-624

[14]. Martyn JB, Moreno RH, Paré PD, Pardy RL. Measurement of inspiratory muscle performance with incremental threshold loading. Am Respir Dis. 1987;135(4):919-23.

[15]. YdforsM, Hughes MC, Laham R, Schlattner U, Norrbom J, Perry CGR. Modelling in vivo creatine/phosphocreatine in vitro reveals divergent adaptations in human muscle mitochondrial respiratory control by ADP after acute and chronic exercise.J. Physiol. 2016; 594, 3127-3140.

[16]. Fleiss JL, Cohen J. The equivalence of weighted kappa and the intraclass correlation coefficient as measures of reliability. EducPsycholMeas. 1973;33(3):613-9.

[17]. Shoukri MM, Asyali MH, Donner A. Sample size requirements for the design of reliability study: review and new results. Stat Meth Med Res 2004;13(4):251-271.

[18]. Bland JM, Altman DG. Measuring agreement in method comparison studies. Statistical Methods in Medical Research. 1999;8(2):135-60. 
[19]. Silva PE, de Carvalho KL, Frazão M, Maldaner V, Daniel CR, Gomes-Neto M. Assessment of Maximum Dynamic Inspiratory Pressure. Respiratory Care October 2018, 63 (10) 1231-1238.

[20]. Romer LM, Polkey MI. Exercise-induced respiratory muscle fatigue: implications for performance. J Appl Physiol 2008;104(3):879-888.

[21]. Arend M, Kivastik J, Ma"estu J. Maximal inspiratory pressure is influenced by intensity of the warm-up protocol. Respir PhysiolNeurobiol2016; 230:11-15.

[22]. Minahan C, Sheehan B, Doutreband R, Kirkwood T, Reeves D, Cross T. Repeated-sprint cycling does not induce respiratory muscle fatigue in active adults: measurements from the Powerbreathe ${ }^{\circledR}$ inspiratory muscle trainer. J Sport Sci Med 2015;14(1):233-238.
[23]. Johnson BD, Babcock MA, Suman OE, Dempsey JA Exercise-induced diaphragmatic fatigue in healthy humans. J Physiol1993; 460:385-405.

[24]. Fry DK, Pfalzer LA, Chokshi AR, Wagner MT, Jackson ES Randomized control trial of effects of a 10 -week inspiratory muscle training program on measures of pulmonary function in persons with multiple sclerosis. J Neurol Phys Ther 2007;31, December (4):162-72.

\section{FIGURES}

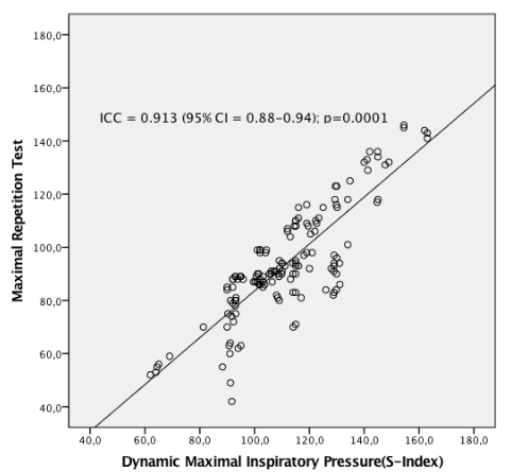

Fig. 1. Interclass correlation coefficient between maximum repetition and dynamic maximum inspiratory pressure. Data are expressed as values, confidence interval and $p$ value.

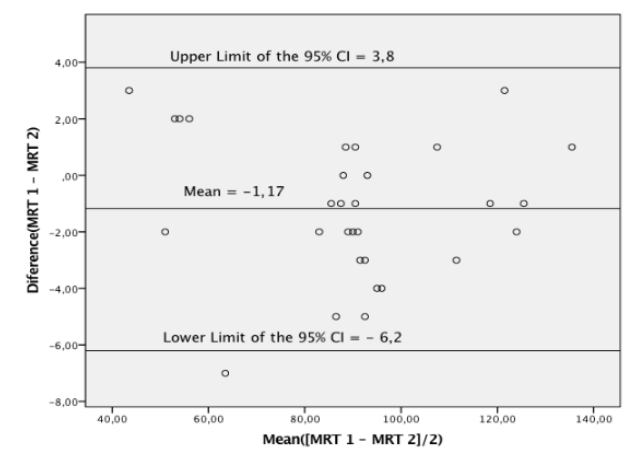

Fig. 3. Precision analysis between the first and second evaluation of the BlandAltman method. Data are expressed as means and confidence interval.

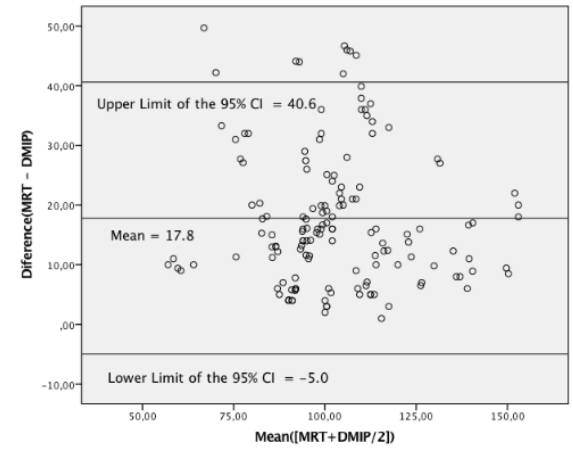

Fig. 2. Precision analysis between the two variables by the Bland-Altman method Data are expressed as mean and confidence interval.

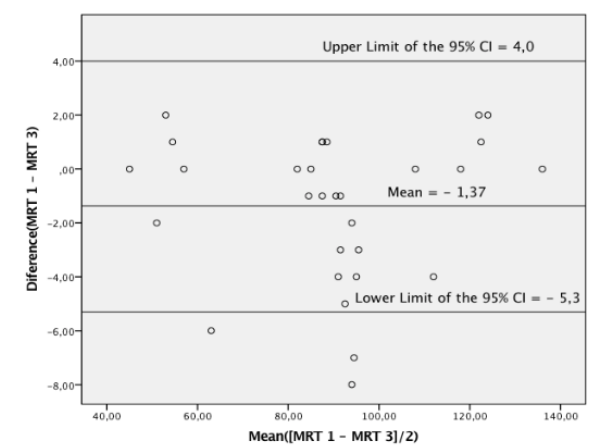

Fig. 4. Precision analysis between the first and third evaluation of the BlandAltman method. Data are expressed as means and confidence interval. 
Is It Possible To Test The Maximum Repetition For The Inspiratory Muscle? Feasibility, Reliability and a Comparison with the Maximum Dynamic Inspiratory Pressure

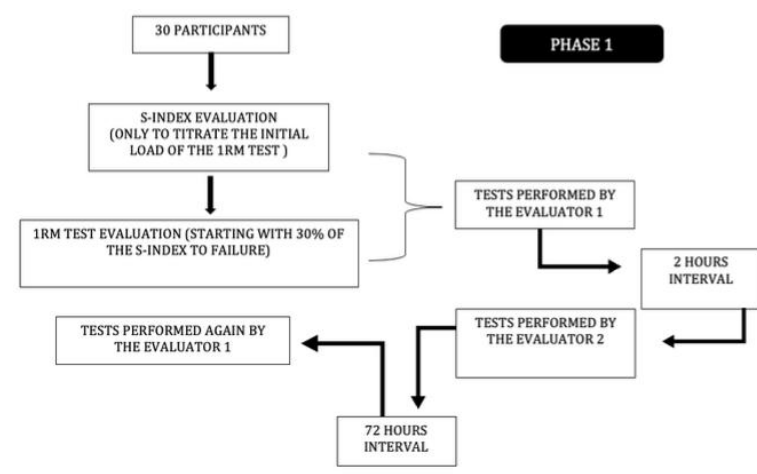

Fig. S1. First phase of the evaluations, which consisted of testing the reliability of the $1 M R$ test.

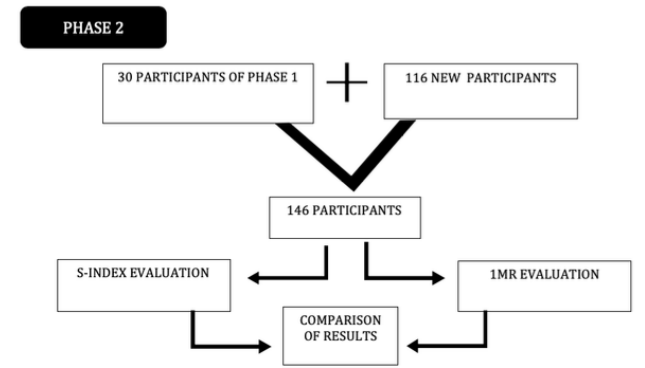

Fig. S2. second phase of the evaluations, which consisted of comparing the 1RM test with the S-Index.

\section{TABLES}

Table 1- General data of the sample of evaluated individuals.

\begin{tabular}{lcc}
\hline Variables & Total sample $(\mathbf{n}=\mathbf{1 4 6})$ & p value \\
Mean age (SD) & $36(9)$ & \\
Gender $\mathrm{n}(\%)$ & & \\
$\quad$ Male & $106(72,6)$ & \\
Female & $40(24,7)$ & \\
Mean BMI(SD) & $26,4(4,6)$ & \\
AverageHeight (SD) & $1,69(0,09)$ \\
Comorbidities, $\mathrm{n}(\%)$ & & \\
Systemic arterial hypertension & $10(6,8)$ & \\
Diabetes Mellitus & $2(1,4)$ & \\
Failuredetectionform, n (\%) & $115(78,8)$ & $<0,001$ \\
Ineffectivetriggering & $31(21,2)$ \\
$\quad$ Vital capacity & $111,8(19,7)$ & \\
Mean S-Index(SD) & $94(20,7)$ & \\
Mean MR (SD) & $17,75(15,9 \%)$ &
\end{tabular}

$\mathrm{BMI}=$ Body mass index, $\mathrm{SD}=$ standard deviation, $\mathrm{S}$-Index $=$ Maximum dynamic inspiratory pressure, $\mathrm{MR}=$ Maximum repetition.

Table 2 - ICC values for intra-examiner and inter-examiner MR test

\begin{tabular}{lccc}
\hline Variables & Value & CI 95\% & p value \\
ICC for the intra-examiner & 0,996 & $0,993-0,998$ & $<0,001$ \\
ICC for the inter-examiner & 0,997 & $0,994-0,999$ & $<0,001$
\end{tabular}

$\mathrm{MR}=$ Maximum repetition, $\mathrm{ICC}=$ interclass correlation coefficient. 